\title{
Convolution Neural Network Architectures for Motor Imagery EEG Signal Classification
}

\author{
Nagabushanam Perattur, KITS, India \\ S. Thomas George, KITS, India \\ D. Raveena Judie Dolly, KITS, India \\ Radha Subramanyam, KITS, India \\ (iD) https://orcid.org/0000-0003-4817-3683
}

\begin{abstract}
This paper has made a survey on motor imagery EEG signals and different classifiers to analyze them. Resolution for medical images like CT, MRI can be improved using deep sense CNN and improved resolution technology. Drowsiness of a student can be analyzed using deep CNN and it helps in teaching, assessment of the student. The authors have proposed 1D-CNN with 2 layers and 3 layers architecture to classify EEG signal for eyes open and eyes closed conditions. Various activation functions and combinations are tried for 2-layer 1D-CNN. Similarly, various loss models are applied in compile model to check the CNN performance. Simulation is carried out using Python 2.7 and 1D-CNN with 3 layers show better performance as it increases number of training parameters by increasing number of layers in the architecture. Accuracy and kappa coefficient increase whereas hamming loss and logloss decreases by increasing number of layers in CNN architecture.
\end{abstract}

\section{KEYWORDS}

Artifacts, EEG, Epilepsy, Motor Imagery, Seizures, Sleep Scoring

\section{INTRODUCTION}

EEG signals may be affected by artifacts at the time of recording. Adaptive classifiers with weighted distance nearest neighbor classifiers with auto regressive models, power being the features considered can give better classification performance (M. Sabeti et. al, 2013). Generalized RNN is used to detect prestate seizures in EEG. Ten sub frequency bands are created from EEG, features are extracted using regression neural network and then applied to ten threshold mechanisms for classification (C. Sudalaimani et. al, 2018). Gradient descent, optimization techniques are used in logistic regression and $\beta$ function for sigmoid helps in incrementing accuracy for the identification of malignant or benign tissue presence for cancer analysis (Laila Khairunnahara et. al, 2019).

Section 2 gives an overview on brain related issues, Section 2.1 describes motor imagery classification methods. Section 2.2 explains the availability of different classifiers for EEG, Section 
3 illustrates the proposed architectures for 1D-CNN, Section 4 show the results obtained using python 2.7 and narrates the possibility to increase the performance of CNN, Section 5 concludes the work carried out.

\section{SURVEY OF BRAIN RELATED ISSUES}

\subsection{Motor Imagery Classification}

5-layer CNN extract features and classify motor imagery EEG with left hand and right-hand movements (Zhichuan Tang, et. al, 2016). Conventional methods like SVM with power, SVM with common spatial pattern and SVM with autoregression are used in motor imagery classification. CNN performs better than the conventional classification methods. Combining deep learning with data augmentation for 2-way motor imagery classification (Zhiwen Zhang et. al, 2018). EMD decomposes EEG, mixes their IMFs to form new artificial frames of EEG, applied as inputs to complex morlet wavelets. CNN with wavelet NN helps in obtaining higher classification accuracy. STFT trains EG and give frequency domain representation. CNN and LSTM are deep NN used for EEG motor imagery classification with promising results (ZijianWang et.al, 2017).

Spatial distributions, $\beta$ and $\mu$ rhythms help in imagery activities classification of EEG signals. Gradient descent, MLP are used for training the neural network which may lead to less accuracy, speed of convergence, PSO-GSA attains better accuracy, convergence speed in motor imagery classification (Sajjad Afrakhteh et. al, 2018), (Rahul Kala et. al, 2011). EEG muscular motor imagery is approximated based on RBF, further conventional MLP-NN and asynchronous NN are applied to increase accuracy, speed of control for the EEG classification (I. E. Shepelev et. al, 2018). Unclean EEG filtering, low SNR problems can be addressed using traditional BPNN, however an improved BPNN with weight splitting technique and PSO for appropriately training the low weights help in better motor imagery classification (Long Liu, 2019). Table 1 shows various techniques on motor imagery EEG

Table 1. Various motor imagery EEG aspects and techniques used

\begin{tabular}{|l|l|l|l|l|}
\hline \multicolumn{1}{|c|}{ Authors } & \multicolumn{1}{|c|}{ Year } & \multicolumn{1}{|c|}{ Type of Disease } & \multicolumn{1}{c|}{ Approaches } & \multicolumn{1}{c|}{ Achievement } \\
\hline Sunny T. D. et. al & 2016 & $\begin{array}{l}\text { Motor imagery } \\
\text { classification }\end{array}$ & $\begin{array}{l}\text { Bayesian spatio-spectral } \\
\text { filters }\end{array}$ & $\begin{array}{l}\text { Classification } \\
\text { performance }\end{array}$ \\
\hline Rami Alazrai et. al & 2018 & $\begin{array}{l}\text { Decoding finger } \\
\text { movements-EEG }\end{array}$ & Choi William, quadratic T-F & 2-way classification \\
\hline Akara Supratak et. al & 2017 & Sleep stage scoring & CNN, bidirectional LSTM & Accuracy, F1 score \\
\hline Nicola Michielli et. al & 2019 & $\begin{array}{l}\text { Sleep stage } \\
\text { classification }\end{array}$ & Cascaded LSTM RNN & $\begin{array}{l}\text { Neuro cognitive } \\
\text { performance }\end{array}$ \\
\hline Arnaud Sors et. al & 2017 & $\begin{array}{l}\text { Sleep stage scoring } \\
\text { CNN }\end{array}$ & $\begin{array}{l}\text { Cohort study, class } \\
\text { wise patterns }\end{array}$ \\
\hline Irene Sturm et. al & 2016 & $\begin{array}{l}\text { Motor imagery } \\
\text { classification }\end{array}$ & $\begin{array}{l}\text { Layer-wise propagation } \\
\text { complex perception }\end{array}$ \\
\hline
\end{tabular}

\subsubsection{Inferences}

- Motor imagery classification with left hand, right hand movement can be done using conventional methods, CNN.

- Gradient descent, MLP are conventional training NN methods, PSO-GSA can give better convergence speed in motor imagery classifications. 
- $\quad$ MLP-NN, asynchronous NN can increase accuracy, speed of control in muscular motor imagery EEG signal classification.

- Improved BPNN with weight splitting and PSO for appropriate weights training achieve better accuracy for motor imagery EEG classification.

- Deep EEG features and classification for motor imagery can be done using STFT, CNN and LSTM.

\subsection{Types of Classifiers for EEG}

Cerebral disorders like crueltzfeldt-Jakob disease is analyzed using SVM for better classification accuracy after dimensionality reduction using PCA (Valeria sacca et. al, 2018, Franziska Matth"aus, et. al, 2009). Brain atlases from ADNI database is collected for gray matter classification. LONI and AAL atlases are used. PCA used for dimensionality reduction and SVM for classification (Yousra Asim et. al, 2017). DWT is applied to extract features like mean, median, skewness, relative wave energy, variance, energy. SVM based on radial basis function, KNN, naïve bayes machine learning methods are applied on ictal, inter-ictal data from bonn university database for eyes open and closed conditions to obtain higher accuracy (Asmaa Hamada et. al, 2018).

Cerebral micro bleeding is a serious issue in EEG which further leads to strokes like dementia, inter-cerebral hemorrhage and death. So, it is to be treated well in advance for the patient to have healthy life. 9-layer CNN with 6-layers of convolution layers and 3-layers of fully connected layers help in better identification of micro bleeding (ShuihuaWang et. al, 2018), (Jian-min Liu et. al, 2018). Super resolution (SR) CNN, fast SRCNN and deep sense SRCNN can be used to improve resolution of CT and MRI images (ShuaifangWei et. al, 2018). Deep CNN can be used to detect the drowsiness state of person by reducing the complexity in manual feature extraction and effects of interference in environment (Gang Zhao et. al, 2018), (Nabil M. Hewahi et. al, 2017). This analyzes the learning state of a student and helps in teaching and assessment of the person. Computer aided designs (CAD) to identify abnormalities or normal, preictal, seizures from EEG using machine learning techniques are carried out (U. Rajendra Acharya et. al, 2017). A 13-layer deep CNN is used to diagnosis epilepsy. Table 2 shows various classifiers on EEG signals.

Table 2. Various classifiers used on EEG signals

\begin{tabular}{|l|l|l|l|l|}
\hline \multicolumn{1}{|c|}{ Authors } & \multicolumn{1}{|c|}{ Year } & \multicolumn{1}{c|}{ Type of Classifier } & \multicolumn{1}{c|}{ Disease } & \multicolumn{1}{c|}{ Achievement } \\
\hline $\begin{array}{l}\text { KentA.Kiehl } \\
\text { et. al }\end{array}$ & 2013 & $\begin{array}{l}\text { KNN - non linear } \\
\text { discriminative method }\end{array}$ & $\begin{array}{l}\text { Schizophrenia- functional } \\
\text { connectivity }\end{array}$ & $\begin{array}{l}\text { FNC features- } \\
\text { schizophrenia }\end{array}$ \\
\hline Tianyi Zhou et. al & 2018 & $\begin{array}{l}\text { Multifeatured fusion, SVM } \\
\text { linear classifier }\end{array}$ & $\begin{array}{l}\text { Autism spectrum disorder } \\
\text { (ASD) }\end{array}$ & Accuracy 91.38\% \\
\hline $\begin{array}{l}\text { Raja Majid Mehmood } \\
\text { et. al }\end{array}$ & 2017 & $\begin{array}{l}\text { SVM, KNN, LDA, DL, } \\
\text { random forest }\end{array}$ & Emotion recognition & $\begin{array}{l}\text { Hjorth parameters, } \\
\text { spectral powerband }\end{array}$ \\
\hline $\begin{array}{l}\text { U Rajendra Acharya } \\
\text { et. al }\end{array}$ & 2018 & $\begin{array}{l}\text { DL-Convolutional neural } \\
\text { network }\end{array}$ & Depression screening - EEG & $\begin{array}{l}\text { Depression severity } \\
\text { index }\end{array}$ \\
\hline $\begin{array}{l}\text { Rakesh Ranjan et. al } \\
\text { Rakn }\end{array}$ & 2018 & Fuzzy neural network & $\begin{array}{l}\text { Detect K-complex in sleep } \\
\text { EEG }\end{array}$ & $\begin{array}{l}\text { Neurological, } \\
\text { Mental disorders }\end{array}$ \\
\hline
\end{tabular}

\subsubsection{Inferences}

- Fractal dimensions, AR models and power as features, adaptive weighted distance based nearest neighbor classifiers can give better performance.

- $\quad$ PCA, SVM can help in detecting cerebral disorders like Crueltzfeldt-Jakob disease. 


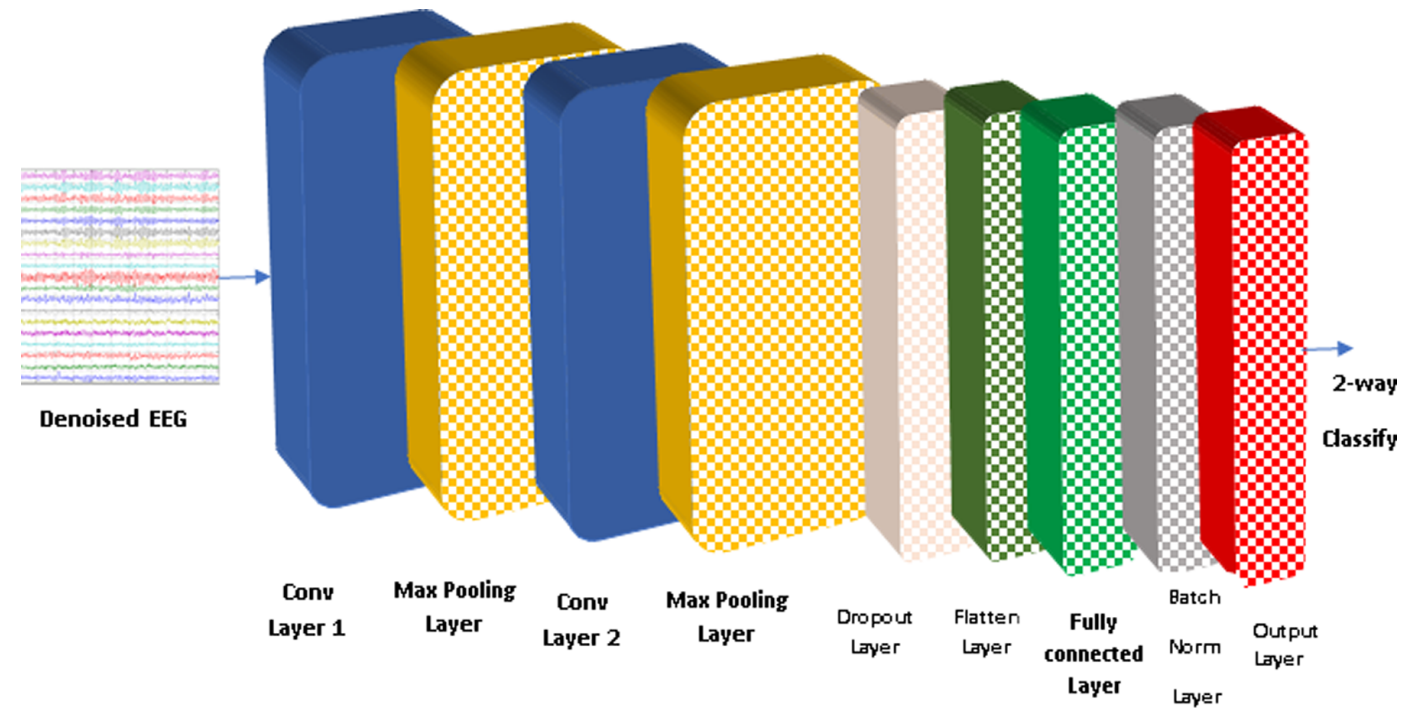

\section{Feature Extraction}

\section{Classification}

- Gray matter, glucose content in each brain region can be classified for ADNI database atlases like LONI and AAL.

- 6-layers of convolution and 3-layers of fully connected in CNN can identify micro bleeding in cerebral EEG.

- DWT applied to extract features, machine learning approaches to classify ictal, inter-ictal for eyes open and eyes closed conditions.

\section{PROPOSED CNN ARCHITECTURES}

CNN stands for convolutional neural network. 1D-CNN can be applied on EEG signals and hence we proposed two architectures of 1D-CNN shown below. Figure 1 shows 1D-CNN with 2 layers in which two convolutional layers and each followed by max pooling layer are used. Max pooling layer applies the filtering process and converts it to lower dimensions, which represents a single value for $3 \times 3$ or $5 \times 5$ filter used for pooling process. Feature extraction is carried out and then applied to dropout layer to drop all the less dominant neurons and hence reduce the size. Flattened layer, fully connected layer further help in increasing number of training parameters to give best results. Batch norm layer is used in further stage to decrease the size. Output layer is dense layer and two-way classification is carried out. Figure 2 show 1D-CNN with 3 layers where 3 convolution layers in appropriate flow is used to obtained better accuracy and to increase the classification performance.

\section{RESULTS AND DISCUSSION}

Motor imagery EEG is collected from open source in which eyes closed and eyes open conditions are the movements/motor considered while capturing EEG. It is filtered to remove any minute noise 
Figure 2. 1D-CNN with 3 layers (Model 2)

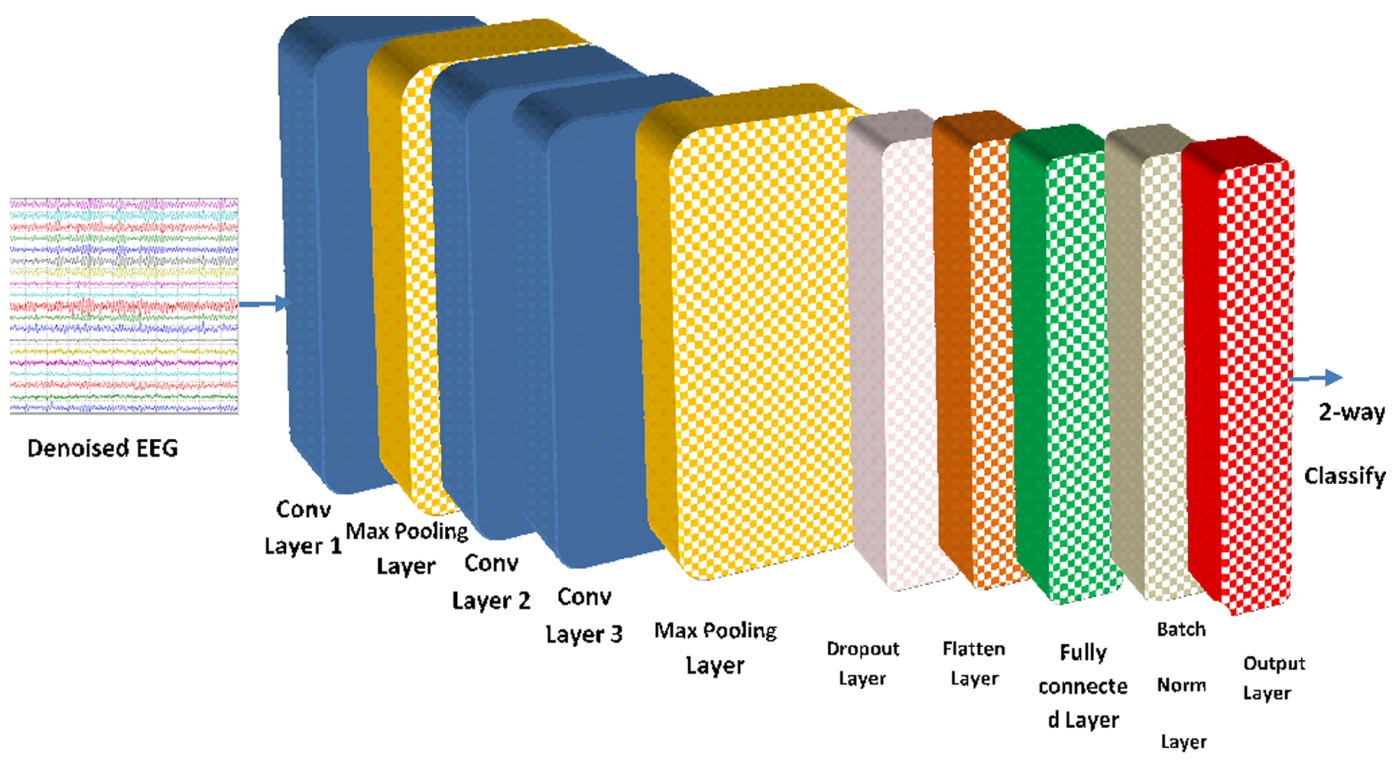

\section{Feature Extraction}

\section{Classification}

and denoised EEG is applied to 1D-CNN with 2 layers and 1D-CNN with 3 layers shown in section3. Different combinations of activation functions, loss models are applied to convolutional layers. Table 3 shows the performance of 1D-CNN with two layers when different combinations of activation functions are used in convolutional layers. Similarly, Table 4 shows the performance of 1D-CNN with two layers when different combinations of loss models are used in convolutional layers. Table 5 shows the performance of 1D-CNN with 2 layers and 1D-CNN with 3 layers. Hence when number of layers are increased, accuracy of classification improves, losses decreases and kappa coefficient

Table 3. Various activation functions used on 1D CNN

\begin{tabular}{|l|l|c|l|l|l|}
\hline & \multicolumn{5}{|c|}{ (loss='binary_crossentropy', optimizer='adam' - fixing) } \\
\hline \multicolumn{1}{|c|}{ Activation functions } & relu, softmax & relu, relu & \multicolumn{1}{|c|}{ relu, selu } & relu, sigmoid & \multicolumn{1}{c|}{ relu, softplus } \\
\hline \multicolumn{1}{|c|}{$\begin{array}{c}\text { 1D-CNN } \\
\text { trial1 }\end{array}$} & $\begin{array}{c}\text { 1D-CNN } \\
\text { trial2 }\end{array}$ & $\begin{array}{c}\text { 1D-CNN } \\
\text { trial3 }\end{array}$ & $\begin{array}{c}\text { 1D-CNN } \\
\text { trial4 }\end{array}$ & $\begin{array}{c}\text { 1D-CNN } \\
\text { trial5 }\end{array}$ \\
\hline Kappa co & 0.000 & 0.316 & 0.292 & 0.244 & 0.220 \\
\hline Accuracy & 0.510 & 0.660 & 0.647 & 0.623 & 0.610 \\
\hline Sensitivity & 0 & 0.509 & 0.550 & 0.555 & 0.582 \\
\hline specificity & 1 & 0.805 & 0.740 & 0.688 & 0.637 \\
\hline Precision & 0 & 0.378 & 0.417 & 0.437 & 0.467 \\
\hline F1 Score & 0 & 0.434 & 0.474 & 0.489 & 0.518 \\
\hline
\end{tabular}


Table 4. Various Loss models used on 1D CNN

\begin{tabular}{|l|l|l|l|l|l|}
\hline & \multicolumn{5}{|c|}{ 1D-CNN } \\
& (Activation function-relu,relu and Optimizer-Nadam - fixed) \\
\hline \multicolumn{1}{|c|}{ Loss Models } & binary_crossentropy & $\begin{array}{c}\text { mean_squared } \\
\text { error }\end{array}$ & $\begin{array}{c}\text { mean_squared } \\
\text { _logarithmic_error }\end{array}$ & $\begin{array}{c}\text { categorical } \\
\text { hinge }\end{array}$ & logcosh \\
\hline Kappa co & 0.381 & 0.485 & 0.490 & 0.000 & 0.499 \\
\hline Accuracy & 0.691 & 0.743 & 0.745 & 0.5 & 0.750 \\
\hline Sensitivity & 0.567 & 0.576 & 0.706 & 0 & 0.635 \\
\hline specificity & 0.814 & 0.909 & 0.784 & 1 & 0.865 \\
\hline Precision & 0.411 & 0.388 & 0.474 & 0 & 0.423 \\
\hline F1 Score & 0.476 & 0.464 & 0.567 & 0 & 0.508 \\
\hline
\end{tabular}

Table 5. Comparison of 1D CNN-2 layers and 1D CNN-3 layers

\begin{tabular}{|l|l|l|}
\hline \multicolumn{1}{|c|}{ Performance metrics } & \multicolumn{1}{|c|}{$\begin{array}{c}\text { 1D CNN } \\
\text { (2 layers) }\end{array}$} & \multicolumn{1}{c|}{$\begin{array}{c}\text { 1D CNN } \\
\text { (3 layers) }\end{array}$} \\
\hline Accuracy & $\mathbf{7 2 . 2 6}$ & $\mathbf{7 4 . 6 9}$ \\
\hline Logloss & 9.58 & 8.73 \\
\hline JS Score & 0.7226 & 0.74 \\
\hline Kappa coefficient & 0.442 & 0.492 \\
\hline Hamming Loss & 0.277 & 0.253 \\
\hline
\end{tabular}

increases. Hence in 3-layer 1D-CNN number of training parameters increases and learning takes place better and thus accuracy is increased to 74.69 .

\section{CONCLUSION}

EEG is denoised using elliptic filter and denoised EEG is applied to CNN architectures for feature extraction and classification. Two different architectures for $1 \mathrm{D}-\mathrm{CNN}$ is tried in this paper. By increasing number of layers, number of training parameters increases and thereby classification performance improves in terms of accuracy, logloss, hamming loss, kappa coefficient. Various combinations of activation functions are used in convolutional layers and various loss models are used in compile model to check the performance of proposed CNN architecture. 3-layer 1D-CNN helps in better 2-way EEG motor imagery classification. 


\section{REFERENCES}

Acharya, Oh, Hagiwara, Tan, Adeli, \& Subha. (2018). Automated EEG-based Screening of Depression Using Deep Convolutional Neural Network. Computer Methods and Programs in Biomedicine.

Acharya, Oh, Hagiwara, Tan, \& Adeli. (2017). Deep convolutional neural network for the automated detection and diagnosis of seizure using EEG signals. Computers in Biology and Medicine.

Afrakhteh, Mosavi, Khishe, \& Ayatollahi. (2018). Accurate Classification of EEG Signals Using Neural Networks Trained by Hybrid Population-Physic-Based Algorithm. International Journal of Automation and Computing. DOI: .10.1007/s11633-018-1158-3

Antoniades, A., Spyrou, L., Martin-Lopez, D., Valentin, A., Alarcon, G., Sanei, S., \& Took, C. C. (2017). Detection of Interictal Discharges With Convolutional Neural Networks Using Discrete Ordered Multichannel Intracranial EEG. IEEE Transactions on Neural Systems and Rehabilitation Engineering, 25(12), 1534-4320. doi:10.1109/TNSRE.2017.2755770 PMID:28952945

Hewahi, N. M., \& Abu Hamra, E. (2017). A Hybrid Approach Based on Genetic Algorithm and Particle Swarm Optimization to Improve Neural Network Classification. Journal of Information Technology Research, 10(3), 48-68. Advance online publication. doi:10.4018/JITR.2017070104

Jain, Gulati, Qashqari, Cooper, Bradbury, Whitney, \& Otsubo. (2018). Peri-oral myoclonia with absences with multiple facial and upper body myoclonia: Overlap epilepsy syndrome. Journal of Clinical Neuroscience, 54, 137-139.

Jiao, Gao, Wang, Li, \& Xu. (2017). Deep Convolutional Neural Networks for Mental Load ClassiPcation based on EEG Data. Pattern Recognition.

Kala, R., Shukla, A., \& Tiwari, R. (2011). Breast Cancer Diagnosis Using Optimized Attribute Division in Modular Neural Networks. Journal of Information Technology Research, 4(1), 34-47. Advance online publication. doi:10.4018/jitr.2011010103

Kiehl, Arbabshirani, Calhoun, \& Pearlson. (2013). Classification schizophrenia patients resting-state functional network connectivity. Frontiers in Neuroscience. DOI 10.3389/fnins.2013.00133

Kinney-Lang, Yoong, Hunter, Tallur, Shetty, McLellan, Chin, \& Escudero. (2018). Analysis of EEG networks and their correlation with cognitive impairment in preschool children with epilepsy. Epilepsy \& Behavior, 90, 45-56.

Liu, J., \& Yang, M. (2018). Recognition on Images from Internet Street View Based on Hierarchical Features Learning with CNNs. Journal of Information Technology Research, 11(3), 62-74. Advance online publication. doi:10.4018/JITR.2018070105

Mehmood, R. M., Lee, H. J., \& Du, R. (2017). Optimal Feature Selection Deep Learning Ensembles Method Emotion Recognition From Human Brain EEG Sensors. IEEE Access, 5, 2169-3536.

Michielli, Acharya, \& Molinari. (2019). Cascaded LSTM recurrent neural network for automated sleep stage classification using single-channel EEG signals. Computers in Biology and Medicine.

Pang \& Robinson. (2018). Neural mechanisms of the EEG alpha-BOLD anticorrelation. NeuroImage.

Ranjan, Arya, Fernandes, Sravya, \& Jain. (2018). A Fuzzy Neural Network Approach for Automatic K-Complex Detection in Sleep EEG Signal. Pattern Recognition Letters.

Sezer, E., Işik, H., \& Saracoğlu, E. (2010). Employment and Comparison of Different Artificial Neural Networks for Epilepsy Diagnosis from EEG Signals. Journal of Medical Systems, 36(1), 347-362. doi:10.1007/s10916010-9480-5 PMID:20703714

Shepelev, I. E., Lazurenko, D. M., Kiroy, V. N., Aslanyan, E. V., Bakhtin, O. M., \& Minyaeva, N. R. (2018). A Novel Neural Network Approach to Creating a Brain-Computer Interface Based on the EEG Patterns of Voluntary Muscle Movements. Neuroscience and Behavioral Physiology, 48(9), 1145-1157. doi:10.1007/ s11055-018-0679-0

Sors, Bonnet, Mirek, Vercueil, \& Payen. (2017). A convolutional neural network for sleep stage scoring from rawsingle-channel EEG. Biomedical Signal Processing and Control, 42, 107-114. 
Sturm, Lapuschkin, Samek, \& M“uller. (2016). Interpretable Deep Neural Networks for Single-Trial EEG Classification. Journal of Neuroscience Methods.

Sudalaimani, Sivakumaran, Elizabeth, \& Rominus. (2018). Automated detection of the preseizure state in EEG signal using neural networks. Biocybernetics and Biomedical Engineering, 39, 160-175.

Tang, Li, \& Sun. (2016). Single-trial EEG classification of motor imagery using deep convolutional neural networks. Optik, 130,11-18.

Zhou, Kang, Li, \& Han. (2018). EEG-based multi-feature fusion assessment for autism. Journal of Clinical Neuroscience. 